\title{
Levels and Trends of Hypertension at National and Subnational Scale in Iran from 1990 to 2016: A Systematic Review and Pooled Analysis
}

\author{
Sadaf G. Sepanlou, MD, MPH, PhD'; Parinaz Mehdipour, MSc ${ }^{2,3}$; Ali Ghanbari, BSc ${ }^{4}$; Shirin Djalalinia, PhD ${ }^{5}$; Niloofar Peykari, PhD ${ }^{6}$; \\ Amir Kasaeian, PhD ${ }^{1,7,8}$; Nazila Rezaei, MD²; Bagher Larijani, MD'; Reza Malekzadeh, MD'; Farshad Farzadfar, MD, MPH, DSc ${ }^{2 *}$ \\ 'Digestive Diseases Research Center, Digestive Diseases Research Institute, Tehran University of Medical Sciences, Tehran, Iran \\ ${ }^{2}$ Non-Communicable Diseases Research Center, Endocrinology and Metabolism Population Sciences Institute, Tehran University of \\ Medical Sciences, Tehran, Iran \\ ${ }^{3}$ Centre for Epidemiology and Biostatistics, Melbourne School of Population and Global Health, University of Melbourne, Melbourne, \\ Australia \\ ${ }^{4}$ Department of Epidemiology and Biostatistics, School of Public Health, Tehran University of Medical Sciences, Tehran, Iran \\ ${ }^{5}$ Deputy of Research and Technology, Ministry of Health and Medical Education, Tehran, Iran \\ ${ }^{6}$ Iranian Non-communicable Diseases Committee (INCDC), Ministry of Health and Medical Education, Tehran, Iran \\ ${ }^{7}$ Hematology, Oncology and Stem Cell Transplantation Research Center, Research Institute for Oncology, Hematology and Cell \\ Therapy, Tehran University of Medical Sciences, Tehran, Iran \\ ${ }^{8}$ Inflammation Research Center, Tehran University of Medical Sciences, Tehran, Iran \\ ${ }^{9}$ Endocrinology and Metabolism Research Center, Endocrinology and Metabolism Clinical Sciences Institute, Tehran University of \\ Medical Sciences, Tehran, Iran
}

\begin{abstract}
Background: Raised blood pressure is a serious risk factor for several non-communicable diseases (NCDs) in Iran. In this study, we aimed to estimate the mean of systolic blood pressure (SBP) and diastolic blood pressure (DBP) and subsequently, the prevalence of hypertension by sex, age, province, and year in Iran.

Methods: We conducted a systematic review using PubMed, Web of Science, and Scopus databases through December 2017. We also used individual level data from eight national surveys, aggregated data from seven subnational population-based studies, and extracted data reported in 52 published studies. We used a two-stage model including Age-Spatio-Temporal and Gaussian process regression (GPR) to estimate mean systolic and diastolic blood pressure and used a crosswalk model to estimate the prevalence of hypertension by sex, age, province, and year.

Results: The number of hypertensive adults increased in Iran from 1.8 million (882 thousand in women) in 1990 to 13.6 million (7.2 million in women) in 2016. The national age-standardized prevalence of hypertension increased from $8.7 \%$ (7.8-9.7) to $28.8 \%(27.7-30.0)$ in women and from $8.0 \%(7.2-8.9)$ to $24.2 \%(23.1-25.3)$ in men from 1990 to 2016. Mean systolic and diastolic blood pressures show a similar increasing trend.

Conclusion: During the past 27 years, we observed an increase in the age-standardized prevalence and mean levels of blood pressure. If the current trend in levels of blood pressure and prevalence of hypertension continue in the coming years, Iran will not achieve the sixth target of the Global Action Plan by 2020 and the Sustainable Development Goals (SDGs) by 2030.

Keywords: Epidemiology, Hypertension, Iran, Public Health

Cite this article as: Sepanlou SG, Mehdipour P, Ghanbari A, Djalalinia S, Peykari N, Kasaeian A, et al. Levels and trends of hypertension at national and subnational scale in iran from 1990 to 2016: A systematic review and pooled analysis. Arch Iran Med. 2021;24(4):306-316. doi: 10.34172/aim.2021.43
\end{abstract}

Received: October 22, 2020, Accepted: January 27, 2021, ePublished: April 1, 2021

\section{Introduction}

High blood pressure is a globally recognized risk factor for all-cause mortality, specifically for mortality and morbidity due to non-communicable diseases (NCDs), mainly cardiovascular diseases. ${ }^{1,2}$ In 2013, the World Health Assembly developed a global action plan, in which evident targets were set to tackle the epidemic of NCDs. ${ }^{3}$ One of the main targets was to achieve a $25 \%$ relative reduction in the prevalence of high blood pressure from 2010 to 2020, in with hypertension defined as systolic blood pressure (SBP) equal to or higher than $140 \mathrm{~mm}$
$\mathrm{Hg}$ or diastolic blood pressure (DBP) equal to or higher than $90 \mathrm{~mm} \mathrm{Hg} .{ }^{3}$ Previous studies have reported a higher burden attributable to high blood pressure in developing countries, where policies are usually not evidence-based, partly due to scarcity of robust data and evidence. ${ }^{1,4}$ The ambitious targets of Sustainable Development Goals (SDGs) for health and well-being, released by the United Nations in 2015 will not be achieved by 2030 in most developing countries if the existing wide knowledge gap is going to continue., ${ }^{5,6}$ The Lancet task force formed in 2018 also focuses on the grave economic burden of NCDs 
and their risk factors if the existing trends are going to continue. ${ }^{7}$

Policies at all levels are the mainstay of all efforts for preventing and tackling NCDs. The contribution of subnational estimates to implementation of cost-effective policies at national and subnational level should not be ignored. In this regard, the aim of this study was to quantify the trend of high blood pressure from 1990 to 2016 at national and provincial levels in Iran.

\section{Materials and Methods}

In this study we aimed to estimate the mean of SBP and DBP and the prevalence of hypertension by sex and age at national level across all provinces of Iran from 1990 to 2016. Hypertension is defined as SBP equal to or greater than $140 \mathrm{~mm} \mathrm{Hg}$ and/or DBP equal to or greater than $90 \mathrm{~mm} \mathrm{Hg}$ or being treated for hypertension. The population covered in this study included both sexes, 13 age groups from 25 to over 85 years (five-year categories), 31 provinces, and 27 years from 1990 to 2016.

For this study, we conducted a comprehensive systematic review of published and unpublished sources of data in both international and national Iranian databases. The adopted methods for systematic review included: defining the data sources and how to reach them, the search strategy for each data source, inclusion and exclusion criteria to select the results of studies and reports, and methods for

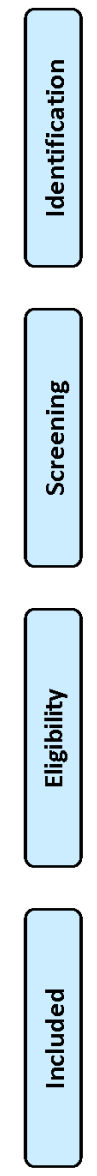

quality assessment, data extraction, and data synthesis.

We searched three groups of data sources: 1) International databases; 2) Iranian databases; 3) Iranian data sources not indexed. We searched for related Mesh terms including all subheadings in international and Iranian databases. The Persian keywords were equivalent to their English words and all possible combinations were considered. The search strategy was developed for MEDLINE, accessed via PubMed, based on an analysis of medical subject headings of key articles identified a priori. We used almost the same search terms for Web of Science and Scopus. We conducted a very sensitive search with broad terms in all three databases. The finalized search terms included were: ("blood pressure" OR hypertension OR prehypertension OR systolic OR diastolic OR "pulse pressure" OR hypertensive) AND (Iran OR Iranian OR Iranians OR Persia OR Persian OR Persians OR “I.R.Iran"). Filters consisted of: 1) Humans and adults; 2) Publication date from 1990/1/1 to 2017/12/31; 3) English and Persian Languages. This search resulted in a total of 2209 hits in PubMed, 1387 hits in Web of Science, and 2,383 hits in Scopus as well as a total of 4453 hits in the overall Iranian databases. A total of 4419 reports were screened by title and abstract, out of which the full-texts of 334 reports were screened. Ultimately 67 studies were selected for inclusion and data extraction. Figure 1 demonstrates the flow chart of study selection.

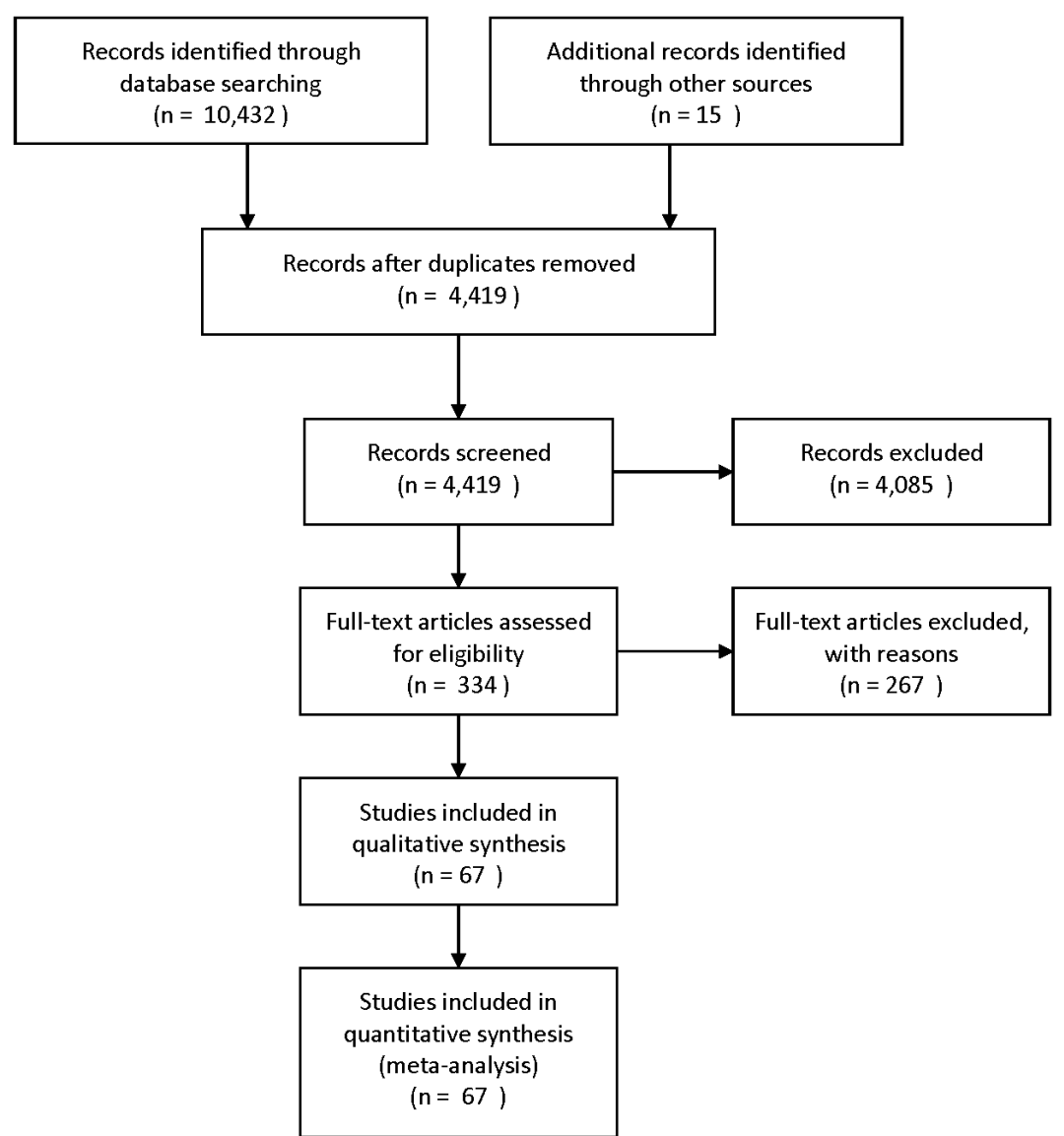

Figure 1. Flow Chart of Study Selection for Systematic Review. 
Only population-based studies were included in which estimates were reported by sex and age, province, year, and either mean SBP and/or DBP. We included all cross-sectional studies and results of the first survey of population-based cohort studies that estimated mean SBP and/or DBP. All national, provincial, district and community studies from January 1990 to December 2017 that reported SBP and/or DBP were included in the study. Studies were included if they were from a representative sample, including from a national, subnational, or community population, and if the data were based on measured (vs. self-reported) blood pressure. If a published article met the inclusion criteria but did not report data by age and sex, we contacted the corresponding author to ask them for the stratified data. Language of publication was restricted to English and Persian. We excluded studies whose sampling method was non-random or their sample was not representative of the Iranian population. We also excluded studies that exclusively reported the prevalence of either hypertension or pre-hypertension. The following criteria were considered in quality assessment of blood pressure measurement: position of patient during blood pressure measurement; whether the arm was measured to determine the correct cuff size; training status of the person taking blood pressure measurement; whether the participants were asked about their activity within the preceding 30 minutes; number of times blood pressure was measured; resting time between each measurement if more than one measurement was made; the time period during which patients was resting before blood pressure measurement; and type of instrument used for measurement.

Two authors (SGS and PM) conducted the search independently. If the two authors could not reach an agreement on including or excluding the data source, FF made the final decision.

We extracted the data reported in 52 population-based studies found in international and Iranian databases at provincial level. Study characteristics are demonstrated in Table S1 of Supplementary file 1. Through the review, we identified 7 population-based cohort studies. We obtained their aggregated data through personal communications with principal investigators of these studies and pooled them for statistical analyses. Additionally, we detected eight national surveys in Iran including: seven iterations of NCD Risk Factors Surveillance Surveys in (STEPwise) in 2005, 2006, 2007, 2008, 2009, 2011, and 2016, and one iteration of National Health Survey (NHS) in 2000. The details on stages of systematic review are presented in the Supplementary file 1 (page 6).

We used a Generalized Linear Mixed Model (GLMM) to estimate the sex- (two sexes) and age-specific (13 age categories from 25 to 85 years with 5-year age intervals) mean of SBP and DBP by province (31 provinces) and year (27 years from 1990 to 2016). ${ }^{8}$ In this model, we included years of schooling, wealth index, and urbanization as fixed-effects covariates and province as random effect of GLMM. The predictions and residuals of our data points were extracted from this model to be used later in the Age-Spatio-Temporal Model. This model was reiterated separately for women and men. We standardized all the estimates with Iran national census population of the last year which is 2016 .

We aimed to consider the variation between sex-ageyear-province combinations by socio-economic status measures including years of schooling, wealth index, and urbanization. These variables helped us to improve the final national and sub-national estimates as they have high correlation with health-related measures and risk factors. Years of schooling were measured according to the level of education attainment for each person extracted from the Statistical Center of Iran (Household and Income Survey). This variable varied from 0 to 25 years. We calculated wealth index based on the Household and Income Survey data for each household. We performed a principal component analysis over all the assets and income of each household. Urbanization was calculated according to the population sizes living in the urban areas over total population size for each age-sex-year-province combination ranging from $0 \%$ to $100 \%$. It is worth mentioning that we transformed each variable to a logarithmic scale to obtain a normal distribution if appropriate. Hypertension was defined as SDP or DBP equal to or higher than $140 / 90 \mathrm{~mm} \mathrm{Hg}$, past medical history of hypertension, or taking antihypertensive medications.

We measured the extra variations in age, space, and time by the Age-Spatio-Temporal model on the residuals extracted from the GLMM model. ${ }^{9}$ In this model, we borrowed strengths over year, provinces, and age groups by smoothing the residuals. To do this, we calculated adjacency matrices over age, province and year. Regarding these matrices, the adjacent provinces, years and similar age groups will be more consistent with each other. Since this model considers the information of neighboring provinces and nearby time points and ages, it can improve hypertension estimates compared to the GLMM model. In the final stage, we used the output from the previous stage as a prior for Gaussian process regression (GPR). , $^{8,10,11}$

To address the various population sizes of different studies, we considered the sample size of each study as one of the variance components in the GPR model. The variance in the GPR model includes two parts for taking into account the variance in each study and between studies. The sampling variance measured the variation due to sampling and small numbers of study with the inverse probability of each study sample size. Uncertainty in the measurements due to non-sampling error was captured in the model for each type of data source. These parameters were estimated on the basis of the degree to which a source tends to disagree with the 
other sources. The more a particular source conflicted with other sources, the higher the variance parameter would be and thus, the more uncertain the measurements. We considered the Matern function as the covariance function in GPR. The Matern function determines the amount of borrowing neighborhood correlation in our timespan. It has three parameters that control correlation and smoothness of the time trend. We made all calculations using a hyperparameter in the Bayesian model. The details of these model are described in Supplementary file 1 (page 8).

In the next stage, we used cross-walk to estimate the prevalence of hypertension using a linear mixed model from mean SBP and DBP estimates. We regressed the logarithm of prevalence of hypertension on the previous mentioned covariates in addition to the mean of SBP and the mean of DBP. We used mean SBP and DBP extracted from GPR as independent variables.

Uncertainty was generated considering both crosssectional and over-time uncertainties. This Bayesian GPR model uses sampling and non-sampling errors to capture all the variation in each data source and between data sources. This is the sum of inverse variance of each data source for sampling error and inverse Gamma distribution for non-sampling errors. We used the "Markov Chain Monte Carlo" method to combine the empirical data and model priors and to draw samples $(n=5000)$ from the posterior distributions of model parameters and estimated prevalence. The median, 2.5 percentile, and 97.5 percentile of this distribution were used as point estimates of lower and upper UIs, respectively.

In order to assess the validity of our estimations, we conducted a sensitivity analysis in two stages. ${ }^{12}$ In the first stage, we randomly masked $10 \%$ of our data points and then, we repeated all the models for the remaining 90\% percent of the data. We used four metrics to evaluate the performance of our in-sample validity: root mean square error (RMSE), Root median square error, median relative error, and mean relative error of prediction models. Median relative error and mean relative error are defined as median of values (SBP, and DBP) over its estimates by each model and mean of absolute value of estimates over its estimates, respectively.

In the next stage, we calculated the proportion of data points in our masked data set that fell within the 95\% uncertainty interval of our $90 \%$ withheld data. For DBP, $96.6 \%$ of the $10 \%$ withheld data hold in $90 \%$ prediction of model and its uncertainty. This measure for SBP was $96.75 \%$.

We calculated the percent change in number of adults with hypertension. We decomposed the contribution of increased prevalence, population growth, and change in age structure to the percent change in the number of hypertensive adults. To estimate the contribution of increase in prevalence, we calculated the percent change in number of hypertensives that would occur since 1990 if population remained the same and only prevalence rose to its level in 2016. Conversely, to estimate the contribution of both population growth and change in age structure, we calculated the percent change assuming the prevalence remained constant and population changed from 1990 to 2016. In order to decompose the contribution of change only due to population growth, we assumed that the prevalence of hypertension remained constant and population growth happened with the same age structure. Finally, to estimate the contribution of change in age structure, we assumed that the population and prevalence remained constant and only the age structure in 1990 changed to the age structure in 2016.

\section{Results}

We collected 67 studies and pooled 10324 data points on about 400 thousand adults, aged 25 to over 85 years, recruited in population-based surveys in Iran from 1990 to 2016. The number of data sources for each province and year is demonstrated in the heat map in Supplementary file 1 (Figure S5). We pooled the data of seven iterations of NCD risk factor surveillances at national level, one iteration of National Health Survey, the aggregated data from seven population-based surveys at provincial level, and the data of 52 published studies extracted through the systematic review.

Nationally, the number of hypertensive population increased from 1.8 million (882 thousand women) in 1990 to 13.6 million (7.2 million women) in 2016.

The age-standardized prevalence of hypertension at national level increased from $8.7 \%(7.8-9.7)$ to $28.8 \%$ $(27.7-30.0)$ in women and from $8.0 \%(7.2-8.9)$ to $24.2 \%$ (23.1-25.3) in men between 1990 and 2016 (Figures 2 and 3 and Table S2 in Supplementary file 1).

From 1990 to 2016, the age-standardized mean SBP in women increased from $115.1 \mathrm{~mm} \mathrm{Hg}$ (111.6-118.7) to $123.1 \mathrm{~mm} \mathrm{Hg}$ (121.0-125.3). In men, the mean SBP increased from $119.3 \mathrm{~mm} \mathrm{Hg}(116.2-122.5)$ to 125.6 mm Hg (123.6-127.6) (Figure 2).

The age-standardized mean DBP in women increased from $74.4 \mathrm{~mm} \mathrm{Hg}(72.1-76.8)$ in 1990 to $80.4 \mathrm{~mm} \mathrm{Hg}$ (78.7-82.1) in 2016. In men, the mean DBP increased from $75.8 \mathrm{~mm} \mathrm{Hg}(73.7-77.9)$ to $80.0 \mathrm{~mm} \mathrm{Hg}$ (78.581.6) (Figure 2 and Figure S1 and S2 in Supplementary file 1).

The mean SBP, DBP, and prevalence of hypertension increased steadily by age groups in all years. The steepest slope of increase in prevalence of hypertension was observed among 55-year-old adults in 1990 and 2000 and among 45-year-old adults in 2010 and 2016 in both sexes (Figure 4 and Figure S3 in Supplementary file 1). The abbreviations for provinces are demonstrated in Table S3 of Supplementary file 1.

The age-standardized prevalence of hypertension from 
National age-standardized
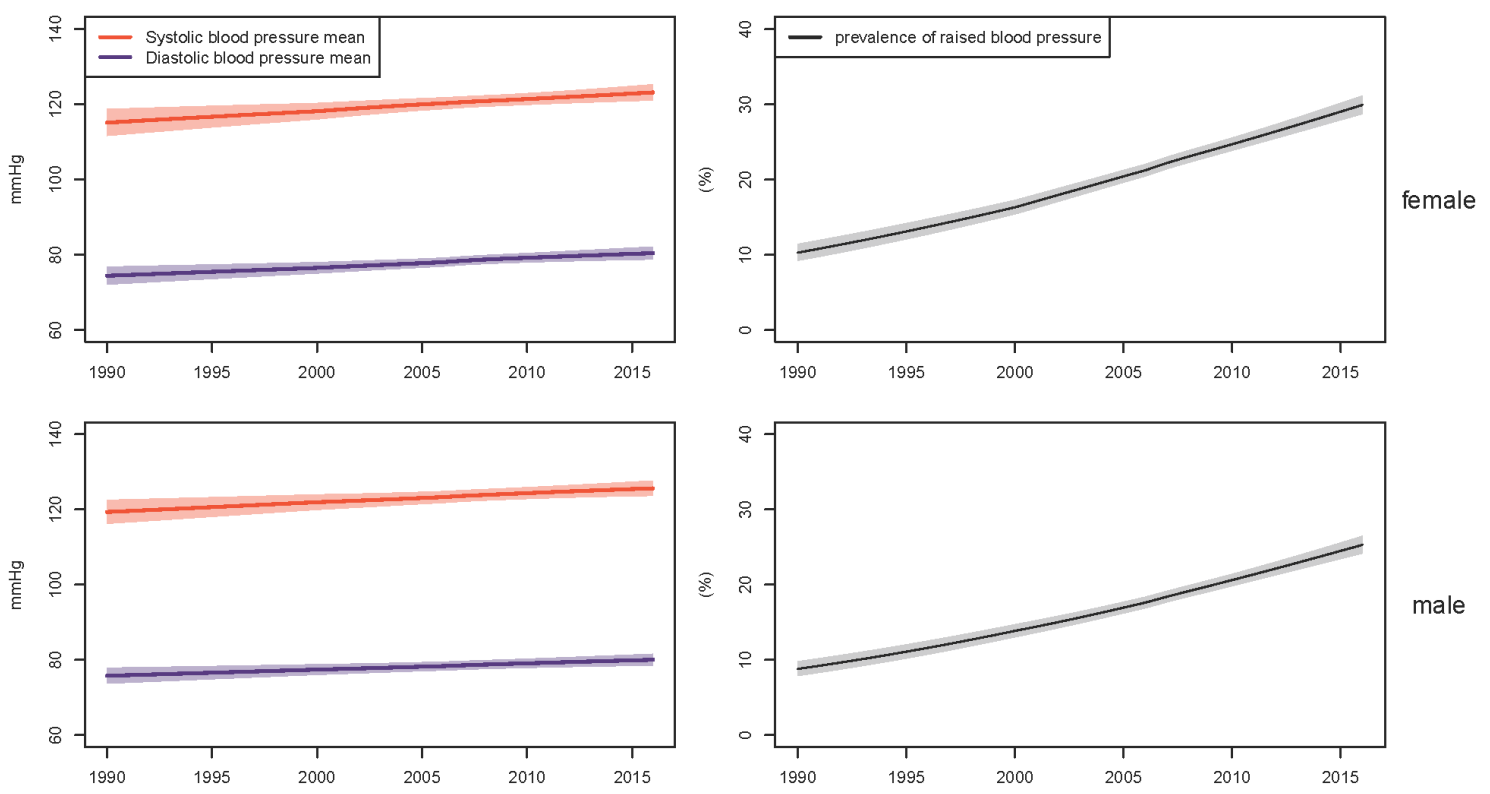

male

Figure 2. Trends in Age-Standardized Mean Systolic and Diastolic Blood Pressure and Raised Blood Pressure by Sex in People Aged 25 Years and Older at National Level from 1990 to 2016. The lines show the posterior mean estimates and the shaded areas show the $95 \%$ UI. See Supplementary file 1 for trends by province.

1990 to 2016 increased by 1.91 folds $(1.51-2.38)$ in women and 1.87 folds (1.46-2.36) in men. The prevalence more than doubled in all provinces and even more than tripled in some provinces in both sexes from 1990 to 2016 (Figure 5 and Figure S4 in Supplementary file 1).

Figure 6 shows the relationship between SBP and DBP with the prevalence of hypertension in men and women. For all levels of mean SBP, the prevalence of hypertension is clearly higher in women than men. This pattern is not observed for DBP.

As mentioned above, the number of adults with hypertension in 2016 increased over seven folds since 1990. In both sexes, $29.4 \%$ of this increase in the number of hypertensive adults was due to the increased agestandardized prevalence, $68.2 \%$ due to population growth, and $2.4 \%$ due to change in age structure (Figure 7 ). The equivalent estimates in women are $27.7 \%$ due to increase in the age-standardized prevalence, $65.7 \%$ due to population growth, and $6.6 \%$ due to change in age structure of the population. In men, the pattern was different. Overall, $31.4 \%$ of the increased number of hypertensive adults was due to the increased age-standardized prevalence and $70.0 \%$ due to population growth. The change in age structure had a negative contribution of $-1.3 \%$ on increase in the number of hypertensive adults. Figure 8 shows that the increase in hypertensive population has occurred mostly in younger adults while the highest increase in number of adults with hypertension is observed in middleaged adults, justifying the non-significant contribution of change in age structure to the increase in the number of hypertensive adults from 1990 to 2016 in Iran.

Across all provinces in 1990, the age-standardized prevalence of hypertension in women ranged from
A) Females
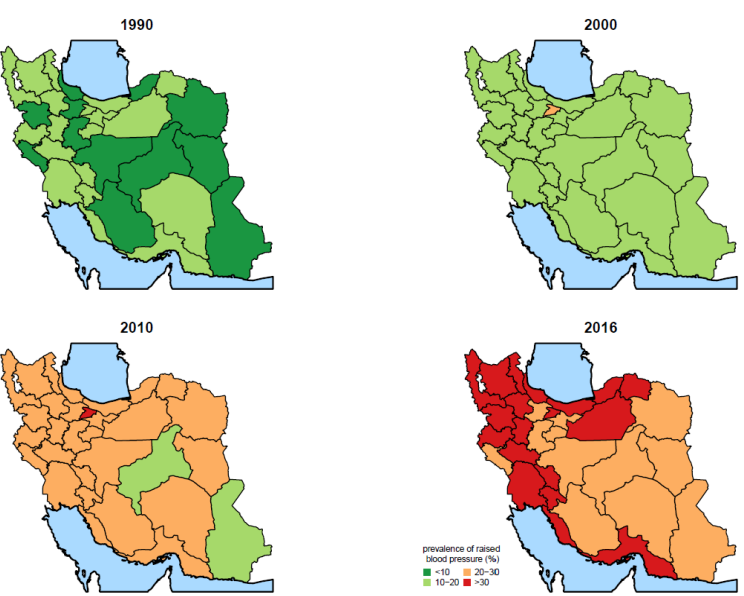

B) Males
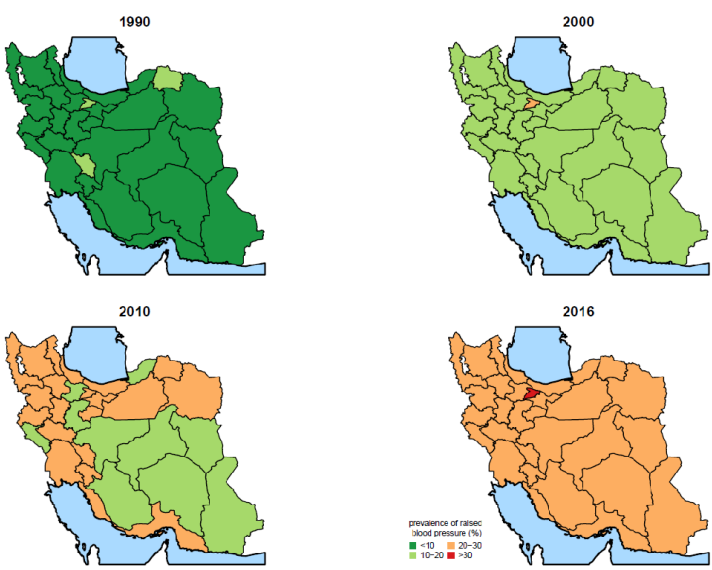

Figure 3. Age-Standardized Prevalence of Raised Blood Pressure by Sex and Province in People Aged 25 Years and Older in 1990, 2000, 2010, and 2016: A) Females, B) Males. Estimates for agestandardized mean systolic and diastolic level by sex in 1990, 2000, 2010, and 2016 are presented in Supplementary file 1. 
A) Female

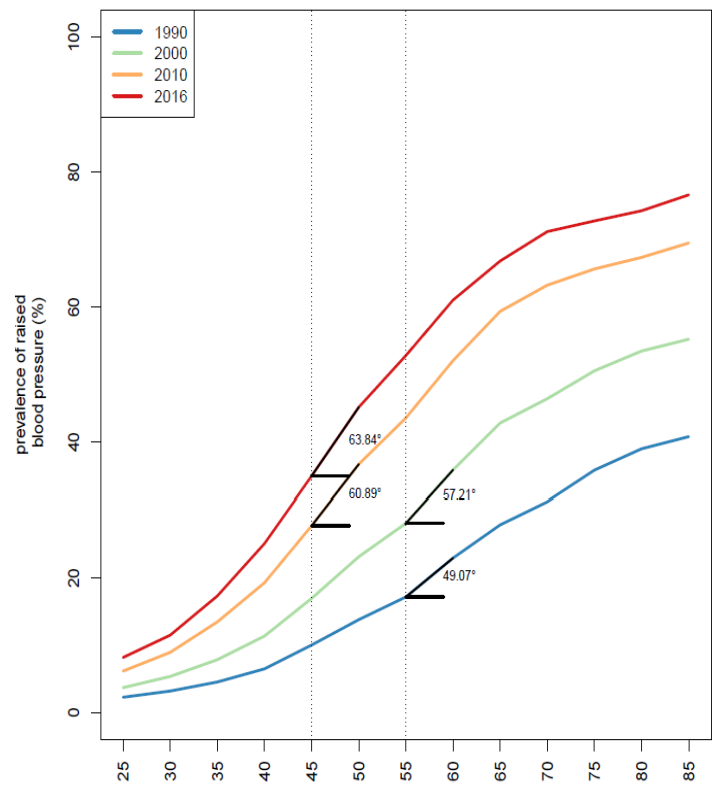

B) Male

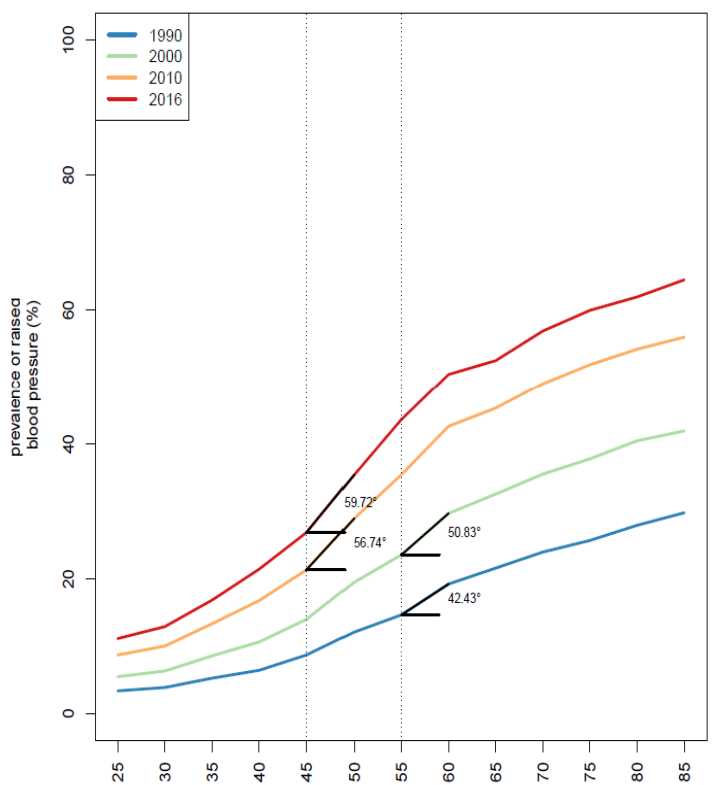

Figure 4. Age Pattern of Raised Blood Pressure Prevalence by Sex in People Aged 25 Years and Older at National Level in 1990, 2000, 2010, and 2016. A) Females; B) Males.

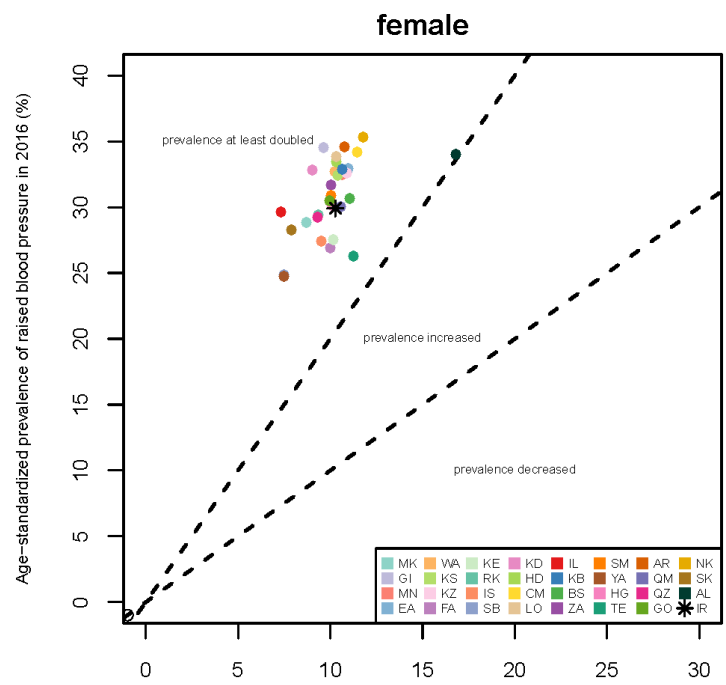

Age-standardized prevalence of raised blood pressure in $1990(\%)$

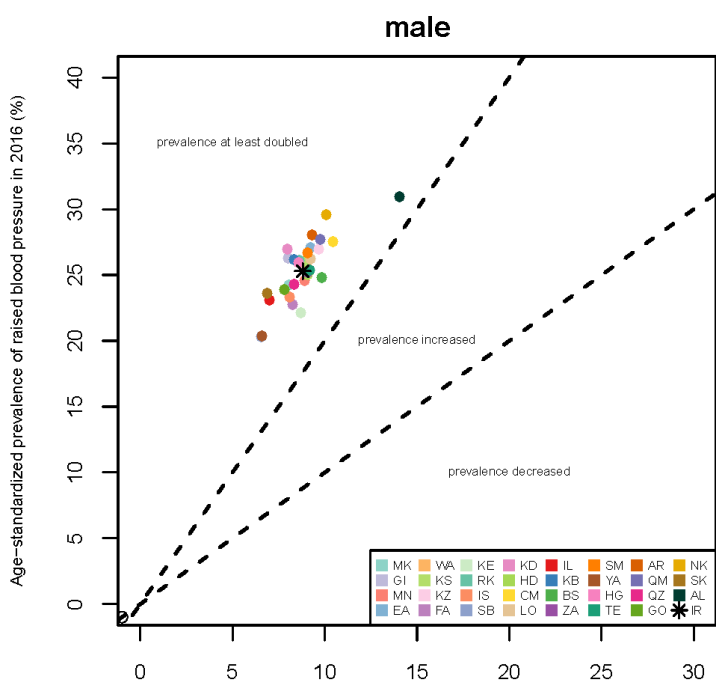

Age-standardized prevalence of raised blood pressure in $1990(\%)$

Figure 5. Comparison of Age-Standardized Prevalence of Raised Blood Pressure in Adults by Sex and by Province between 1990 and 2016.

$7.3 \%(6.4-8.3)$ to $16.8 \%(15.3-18.3)$, and in 2016 , the range was as low as $24.7 \%(23.5 \%-26.0 \%)$ to as high as $35.3 \%(33.7 \%-37.0 \%)$. In men, the age-standardized prevalence across all provinces in 1990 ranged from $6.5 \%$ $(5.7 \%-7.4 \%)$ to $14.0 \%(12.8 \%-15.4 \%)$ and in 2016 , from $20.3 \%(19.2 \%-21.4 \%)$ to $30.9 \%(29.5 \%-32.4 \%)$ (Figure 3 and Figure S1 in Supplementary file 1).

The age pattern of hypertension prevalence was similarly increasing in all provinces in 2016. Yet, there was an evident convergence in hypertension prevalence below 40 years of age and a clear divergence in older age groups (Figure S3 of Supplementary file 1).

Across all provinces, the percent change in age- standardized prevalence from 1990 to 2016 ranged from $102.5 \%$ to $306.0 \%$ in women and from $120.5 \%$ to $243.2 \%$ in men (Figure S4 of Supplementary file 1).

In women, the mean age-standardized SBP among provinces ranged from $119.7 \mathrm{~mm} \mathrm{Hg}(117.6-121.8)$ to $128.4 \mathrm{~mm} \mathrm{Hg}$ (125.6-131.3) in 2016. The mean DBP ranged from $76.8 \mathrm{~mm} \mathrm{Hg}(74.4-79.1)$ to $83.3 \mathrm{~mm} \mathrm{Hg}$ (81.5-85.2). In men, the respective figures for SBP were as low as $121.1 \mathrm{~mm} \mathrm{Hg}(119.0-123.2)$ to as high as 128.7 $\mathrm{mm} \mathrm{Hg}$ (126.7-130.6) in 2016. The lowest observed DBP was $75.5 \mathrm{~mm} \mathrm{Hg}(73.4-77.9)$ and the highest was 83.7 $\mathrm{mm} \mathrm{Hg}(81.8-85.5)$ (Figures S1 and S2 in Supplementary file 1) 

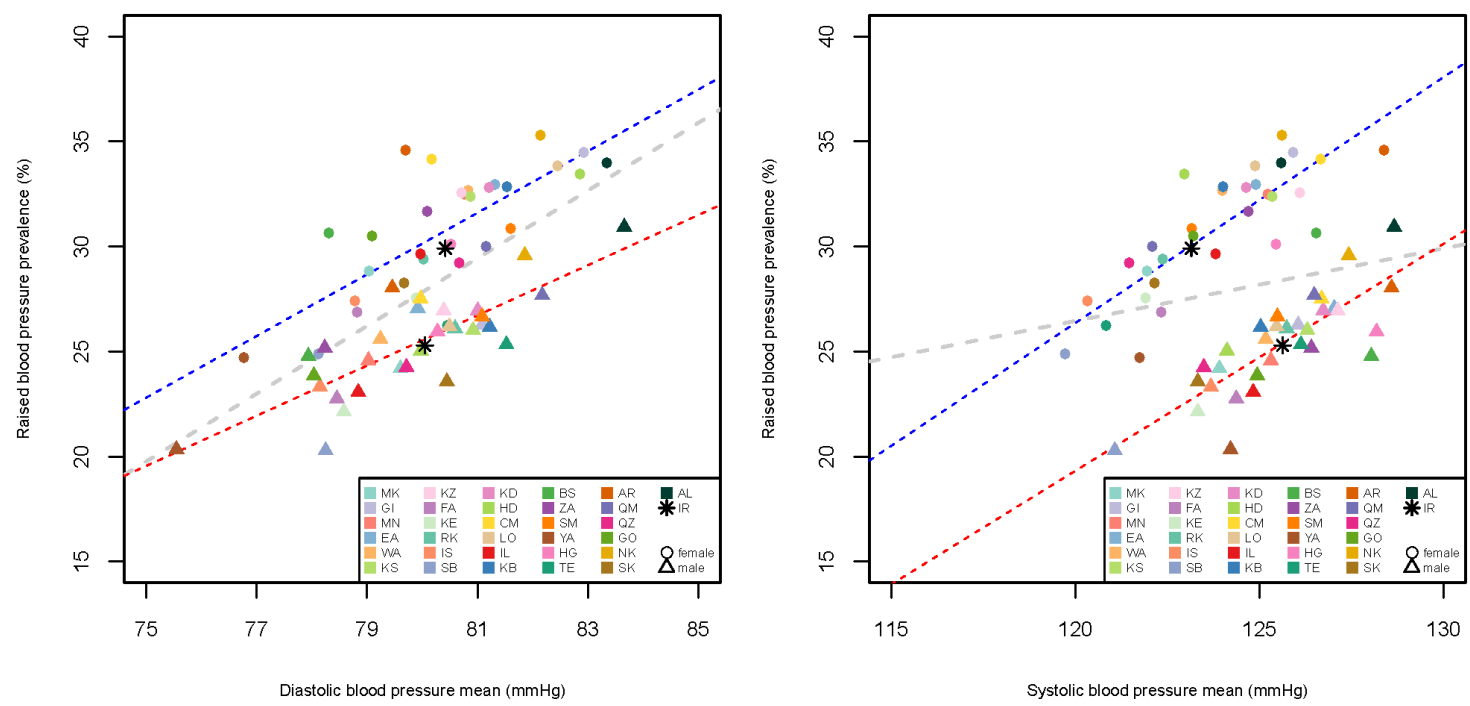

Figure 6. Comparison of Age-Standardized Mean Systolic and Diastolic Blood Pressure, with Prevalence of Raised Blood Pressure in Men and Women Aged 25 Years and Older by Province in 2016.

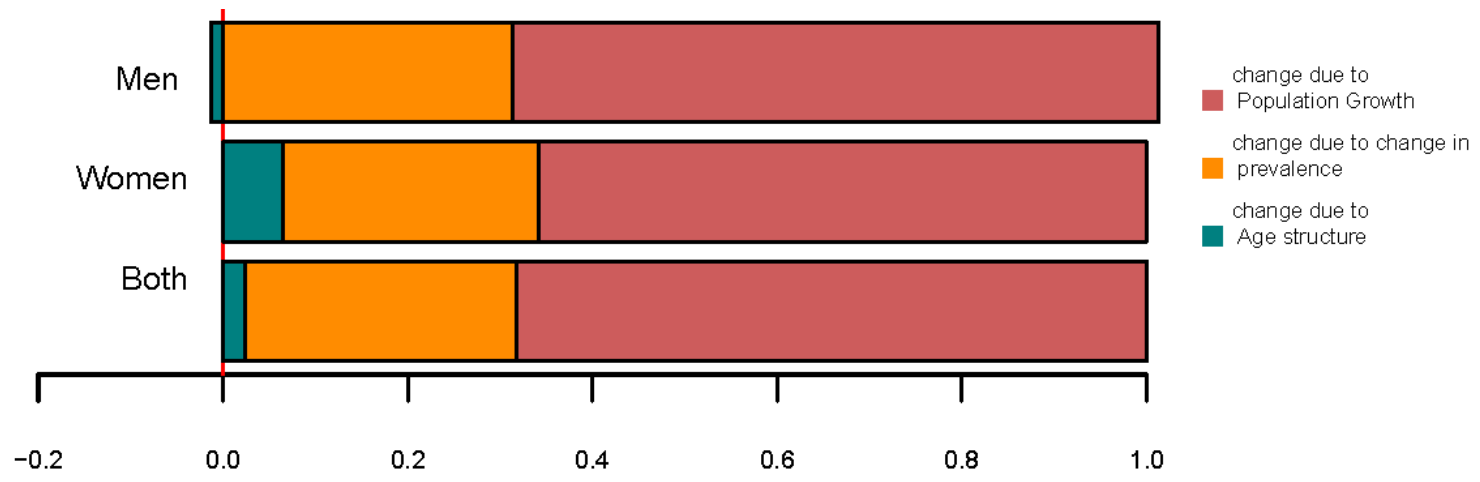

Figure 7. National Percent Change in Number of Adults Aged 25 Years and Older with High Blood Pressure Due to Change in Age-Standardized Prevalence, Population Growth, and Change in Age Structure.

Regarding the trend of mean SBP from 1990 to 2016, the percent change ranged from $1.8 \%(-1.3-5.0)$ to $10.8 \%$ (4.0-18.1) in women. The equivalent results in men were 2.1\% (-1.0-5.3) and 7.4\% (0.7-14.4) (Figures S1 and S2 in Supplementary file 1).

As for the trend of mean DBP across all provinces, the lowest percent change in women from 1990 to 2016 was $4.2 \%(-2.1-11.0)$ and the highest was $12.4 \%(4.3-21.1)$. In men, the equivalent estimates were $2.8 \%$ (-6.0-12.9) and $9.0 \%$ (3.3-14.9) (Figures S1 and S2 in Supplementary file 1).

\section{Discussion}

The results of this study showed a critically steep increase in the age standardized prevalence of hypertension and the number of adults affected in Iran since 1990, mainly due to population growth as well as the increase in age-standardized prevalence. Our results also show a convergence in subnational prevalence implied by the narrowed range of hypertension prevalence across the provinces of Iran in 2016 compared to 1990.

Generally, the prevalence of hypertension was consistently higher in women compared with men across all provinces, age groups, and time intervals. However, the mean levels of SBP and DBP were consistently higher in men compared with women in many provinces and for every level of SBP, the prevalence of hypertension was higher in women than men as demonstrated in Figure 5. This observation implies that anti-hypertensive treatment coverage may be greater in women as treatment was included in the definition of hypertension in the current study.

The age patterns and trends of hypertension prevalence were almost similar in men and women. There was a steady increase in prevalence across the 13 age groups, with the steepest increase in middle-aged adults in both sexes. There is a divergent pattern in the prevalence of hypertension with increasing age in both sexes across the provinces, probably implying the difference in hypertension treatment and control between provinces in older age groups.

The number of adults with high blood pressure has increased by over seven folds. Results of the decomposition analysis show that the increase in the number of adults 

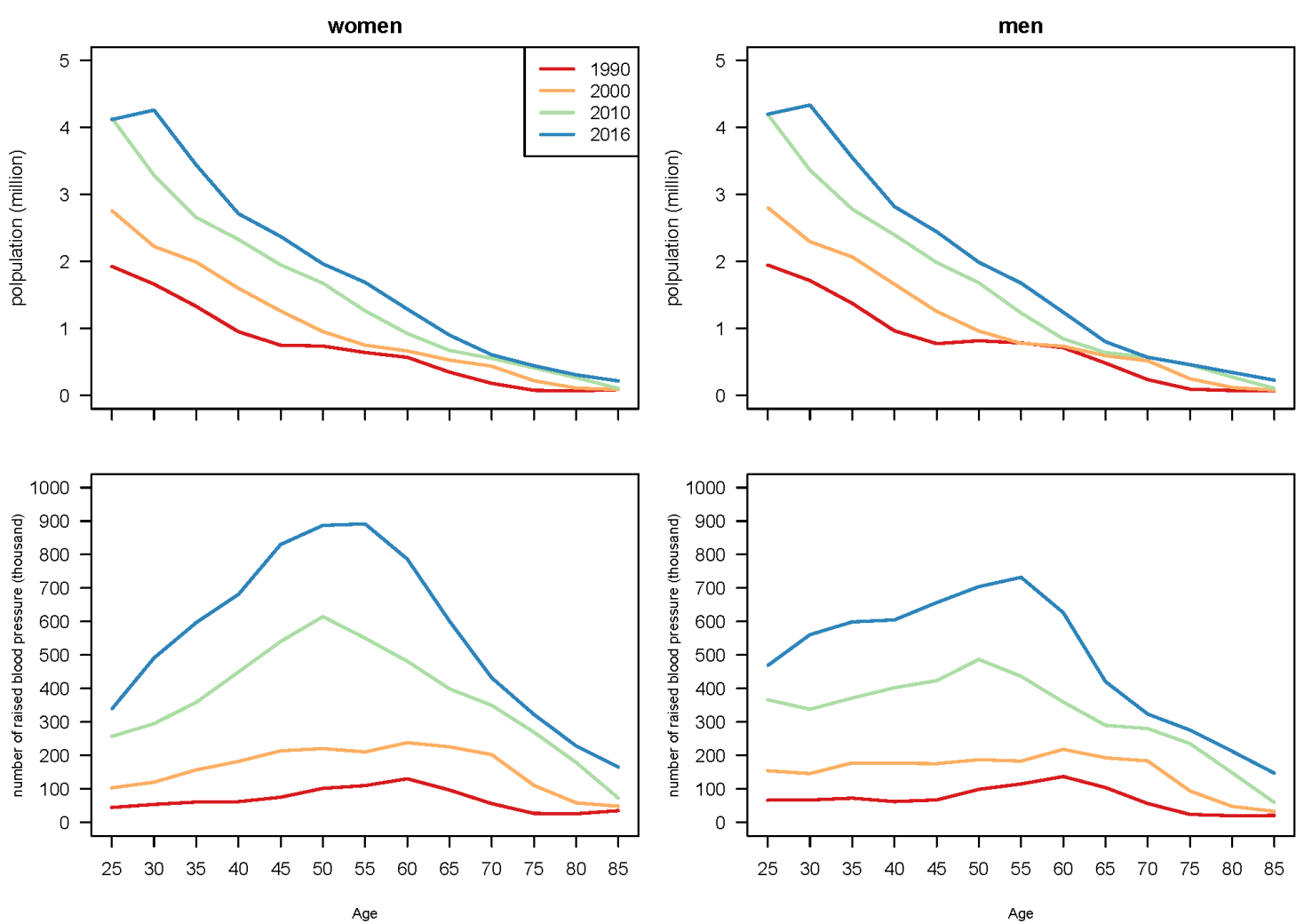

Figure 8. Trend of Population and Number of Adults Aged 25 to Over 85 Years with Raised Blood Pressure from 1990 to 2016.

with hypertension was mostly due to population growth and increase in age-standardized prevalence. Change in age structure had minimal contribution, especially in men where the contribution was negative. Although life expectancy has increased in Iran, the proportion of adults over 50 years in the total population has decreased due to high fertility rates in the late 1970s, 1980s, and early 1990s in Iran. The main increase in the number of hypertensives has occurred in middle-aged adults (Figure 7). This figure also shows that the peak number of the hypertensive adults in the population has shifted from the age of about 65 in 1990 to the age of about 55 in 2016. This may deserve attention as the 10-year shift may have consequences from the viewpoint of health economics and mortality and morbidities arising from the increasing rate of stroke and ischemic heart disease in the population.

The current study provides comprehensive estimations of levels and trends of blood pressure and hypertension prevalence on national and sub-national scales in Iran so far. There are three major alternative studies that have released estimates at the national level in Iran: The NCDRisC study in Imperial College London ${ }^{4}$, the Global Burden of Disease (GBD) Study on burden of high SBP in Institute of Health Metrics and Evaluation, ${ }^{1,2,13}$ and national trend of mean SBP estimated for Iran from 1980 to 2008 by Danaei et al. ${ }^{14}$ Our results show a clearly sharp increase in the prevalence of hypertension (from 10\% to 30\%) between 1990 and 2016 in both sexes, while Ezzati et al reported a clearly decreasing trend from 30\% to $20 \%$ in both sexes between 1975 and $2015 .{ }^{4}$ Yet, the current results on the prevalence of hypertension cannot be completely compared with the reports by Ezzati et al as the definition of hypertension includes anti-hypertensive treatment in our study, which was not included in the definition adopted by the NCD-RisC study. Still, the contrasting trends cannot be easily justified. In GBD 2016, summary exposure value of high SBP was reported with a different definition from that in our study. A decreasing trend was reported, from over $25 \%$ to less than $22 \%$ between 1990 and 2016, which is in evident contrast with the increasing trend we observed in our study.

The mean level of SBP can be compared across all studies. We detected an evidently increasing trend. In NCD-RisC, there was a steady trend in mean SBP of around $120 \mathrm{~mm}$ $\mathrm{Hg}$ in both sexes. In GBD 2015, the age-standardized mean SBP was steady in men (around $124 \mathrm{~mm} \mathrm{Hg}$ ) and slightly decreasing in women (from 124 to $120 \mathrm{~mm} \mathrm{Hg}$ ) between 1990 and 2016. Finally, the results reported by Danaei et al showed an almost U-shaped trend for SBP in men and a slightly decreasing trend in women from 1980 to 2008. As for the mean level of DBP, we observed the same pattern: increasing in our study and a steady trend (around $80 \mathrm{~mm} \mathrm{Hg}$ ) in NCD-RisC.

In short, we observed a steady and sharp increase in SBP and DBP in both sexes since 1990 at the national level in Iran. Other studies showed higher levels in 1990s and lower levels in 2010s compared to our results. To justify this difference, it can be stated that we had more data points than the other studies in 1990s. Otherwise, the discrepancy cannot be justified. 
Our study has certain strengths in comparison with previous studies on the burden of high blood pressure in Iran. We took advantage of a comprehensive systematic review using both published and unpublished sources, in both English and Persian in addition to national surveys, specifically the most recent Non-Communicable Disease Risk Factor Study in 2016. We obtained sexand age-specific aggregated data from several large-scale population-based studies performed in the 1990s and early 2000s. Our data points are evidently much more than the sources based on which previous estimations were made. We used data from studies in which blood pressure was measured and not self-reported. We also excluded studies that exclusively recorded the prevalence of hypertension or pre-hypertension. We assessed the quality of data reported in sources and included only those reports that complied with our strict selection criteria (see Supplementary file 1). Our estimates cover sex and age subgroups, report trends for all years from 1990 to 2016, and most importantly, the estimates are presented on the subnational scale in Iran. We used covariates at subnational level in our estimations. We performed sensitivity analyses to quantify the validity of our models. We computed uncertainty intervals for all of the estimations. The current study is a comprehensive analysis on the level of high blood pressure in Iran.

Our study has certain limitations, as well. In Iran as a developing country, one of the main challenges is data scarcity and low quality of data. We made extensive efforts to clean and harmonize the data from various existing sources. We had data points for at least eight years for all provinces of Iran (eight national studies). Estimates for provinces with low quality and scarce data relied on statistical methodologies. Second, to estimate the prevalence of raised blood pressure, we had to use crosswalks on estimated means of SBP and DBP. Third, we split age groups over 65 years into 5 -year age categories based on the estimates in the last iteration of NCD surveillance survey in 2016. Fourth, various devices and protocols for measuring blood pressure have been used since 1990; this fact may have imposed bias on our estimates.

The drivers of increase in the age-standardized prevalence of hypertension and rising levels of blood pressure were not investigated in this study. Previous evidence demonstrates that high blood pressure is accompanied by several other risk factor of NCDs including high BMI, smoking, and high prevalence of metabolic risk factors of high fasting plasma glucose and high cholesterol. ${ }^{1,13,15,16}$ There is no evidence on a causal relationship of the above-mentioned risk factors with hypertension but reports demonstrate their clustering. There is convincing evidence on the impact of high salt intake and low consumption of fruit and vegetables on hypertension. ${ }^{17}$ National policies to improve diet and physical activity as well as efforts to stop tobacco use are of outmost necessity.

Our study has numerous other policy implications, as well. To address the knowledge gap regarding the burden of NCDs and their risk factors, populationbased national surveys are mandatory for monitoring the trends of risk factors and NCDs as well as evaluating the cost-effectiveness of interventions and improvements in access to primary and secondary health-care on the very provincial scale. This achievement requires integration of health care for NCDs and their risk factors within the health network of Iran. Evidence exists on the impact of training health-care givers at all levels of health care on detection, prevention, and control of NCDs and their risk factors and even the impact of health-care workers density on access to health care and the quality of care. ${ }^{18}$ With the release of new guidelines by the American College of Cardiology and the American Heart Association in November 2017, prevention, detection, evaluation, and treatment of high blood pressure in adults become stricter along with lower thresholds for definition of hypertension and lower treatment goals in primary and secondary prevention. ${ }^{19}$ With the new definitions, the estimated prevalence of hypertension will be much higher.

In conclusions, particularly in Iran, the health network still does not possess the required capacity to tackle with NCDs and their risk factors. ${ }^{20,21}$ Previous reports by Farzadfar et al showed that in 2005, high SBP was responsible for 80 thousand deaths in Iran. ${ }^{22}$ More recently, the results of GBD 2016 demonstrated that over $20 \%$ of all deaths in Iran, which is equivalent to over 80 thousand deaths in both sexes, were attributable to hypertension in 2016 and high blood pressure ranks first among all metabolic, behavioral, and environmental risk factors in Iran. ${ }^{1,13,15}$ Urgent action is required to tackle the burden of NCDs and hypertension as their main risk factor. Inter-sectoral collaborations to drive food markets towards producing healthier foods and implementing taxes on unhealthy food products and tobacco are mandatory. In this regard, the Iranian Non-Communicable Diseases Committee was established to develop an action plan based on the collaboration of policy makers and researchers in multiple sectors. ${ }^{23}$ This plan can be a platform for future improvements of the Iranian health care system in combatting the epidemic of NCDs and their risk factors.

\section{Authors' Contribution}

FF designed and supervised the study. SGS did the comprehensive systematic review, wrote the manuscript, edited the content of manuscript, and discussed the results. PM did entire data preparation, statistical analysis, prepared results, and wrote the manuscript. AG prepared results. SD did data collection through contacting principal investigators of population-based studies. NP did data collection through contacting principal investigators of population-based studies. AK did the original data preparation. NR discussed the results. BL supervised administrative process. RM supervised administrative 
process. The rest of the co-authors were collaborators in our team.

\section{Conflict of Interest Disclosures}

The authors declare that they have no competing interests.

\section{Ethical Statement}

Not applicable.

\section{Funding}

This study was funded by Iran Ministry of Health and Medical Education. Research reported in this publication was also supported by Elite Researcher Grant Committee under award number 958657 from the National Institutes for Medical Research Development (NIMAD), Tehran, Iran. The funding bodies had no role in the design of the study and collection, analysis, and interpretation of data and in writing the manuscript.

\section{Availability of Data and Materials}

The datasets used and/or generated and analyzed during the current study are not publicly available as we do not have the permission to make available the individual level data from national surveys and aggregated data from subnational population-based studies. Data are available from the corresponding author on reasonable request.

\section{Supplementary Materials}

Supplementary file 1. The levels and trends of hypertension at national and sub-national scale in Iran from 1990 to 2016

\section{Acknowledgements}

The authors would like to acknowledge the NASBOD Risk Collaborating Group as co-authors for their substantial contribution to this study. The authors would also like to express their appreciation for partnership of deputy for public health and deputy for research and technology of the Ministry of Health and Medical Education, Islamic Republic of Iran, National Institute for Health Research, and many scholars and experts in related fields. We would also like to express thanks to all of participants and scientific and executive partners of medical sciences universities, who made this experience possible.

NASBOD Risk Collaborating Group consists of the following members, displayed with their affiliations: Fereydoun Azizi (Endocrine Research Center, Research Institute for Endocrine Sciences, Shahid Beheshti University of Medical Sciences, Tehran, Iran), Majid Ghayour-Mobarhan (Metabolic Syndrome Research Center, School of Medicine, Mashhad University of Medical Sciences, Mashhad, Iran), Davood Khalili (Prevention of Metabolic Disorders Research Center, Research Institute for Endocrine Sciences, Shahid Beheshti University of Medical Sciences, Tehran,
Iran), Masoud Khoshnia (Golestan Research Center of Gastroenterology and Hepatology, Golestan University of Medical Sciences, Gorgan, Iran), Jalil Kouhpayehzadeh (Center for Educational Research in Medical Sciences (CERMS), Iran University of Medical Sciences, Tehran, Iran), Shahin Merat (Liver and Pancreaticobiliary Disease Research Center, Digestive Diseases Research Institute, Tehran University of Medical Sciences, Tehran, Iran), Ali Mirzazadeh (Department of Epidemiology and Biostatistics, University of California San Francisco, San Francisco, United States), Kazem Mohammad (Department of Biostatistics and Epidemiology, School of Public Health, Tehran University of Medical Sciences, Tehran, Iran), Noushin Mohammadifard (Hypertension Research Center, Cardiovascular Research Institute, Isfahan University of Medical Sciences, Isfahan, Iran), Iraj Nabipour (The Persian Gulf Marine Biotechnology Research Center, The Persian Gulf Biomedical Sciences Research Institute, Bushehr University of Medical Sciences, Bushehr, Iran), Mahboubeh Parsaeian (Department of Biostatistics and Epidemiology, School of Public Health, Tehran University of Medical Sciences, Tehran, Iran), Akram Pourshams (Digestive Diseases Research Center, Digestive Diseases Research Institute, Tehran University of Medical Sciences, Tehran, Iran), Hossein Poustchi (Liver and Pancreaticobiliary Disease Research Center, Digestive Diseases Research Institute, Tehran University of Medical Sciences, Tehran, Iran), Nizal Sarrafzadegan (Isfahan Cardiovascular Research Center, Cardiovascular Research Institute, Isfahan University of Medical Sciences, Isfahan, Iran).

\section{References}

1. Gakidou E, Afshin A, Abajobir AA, Abate KH, Abbafati C, Abbas KM, et al. Global, regional, and national comparative risk assessment of 84 behavioural, environmental and occupational, and metabolic risks or clusters of risks, 1990-2016: a systematic analysis for the Global Burden of Disease Study 2016. Lancet. 2017;390(10100):1345-422. doi: 10.1016/s0140-6736(17)32366-8.

2. Forouzanfar MH, Liu P, Roth GA, Ng M, Biryukov S, Marczak L, et al. Global Burden of Hypertension and Systolic Blood Pressure of at Least 110 to $115 \mathrm{~mm} \mathrm{Hg}, 1990-2015$. JAMA. 2017;317(2):165-82. doi: 10.1001/jama.2016.19043.

3. WHO. Global action plan for the prevention and control of Non-Communicable Diseases 2013-2020. Accessed Feb 14, 2021. Available from: http://apps.who.int/iris/ bitstream/10665/94384/1/9789241506236_eng.pdf?ua=1.

4. Zhou B, Bentham J, Di Cesare M, Bixby H, Danaei G, Cowan MJ, et al. Worldwide trends in blood pressure from 1975 to 2015: a pooled analysis of 1479 population-based measurement studies with 19.1 million participants. Lancet. 2017;389(10064):37-55. doi: 10.1016/ s0140-6736(16)31919-5.

5. WHO. Global Status Report on non-communicable diseases. 2014. Geneva. Available from: https://apps.who.int/iris/ bitstream/handle/10665/148114/9789241564854_eng. jsessionid=181D8B8FE504145E49c96E0672639FD7? sequence $=1$

6. United Nations. Transforming our world: the 2030 agenda for sustainable development. 2015. New York. Available from: https:// sustainabledevelopment.un.org/content/documents/21252030\%20 Agenda $\% 20$ for $\% 20$ Sustainable\%20Development $\% 20$ web.pdf. 
7. The Lancet Taskforce on NCDs and economics. Available from: https://www.thelancet.com/series/Taskforce-NCDs-and-economics.

8. Mohammadi Y, Parsaeian M, Mehdipour P, Khosravi A, Larijani B, Sheidaei A, et al. Measuring Iran's success in achieving Millennium Development Goal 4: a systematic analysis of under-5 mortality at national and subnational levels from 1990 to 2015. Lancet Glob Health. 2017;5(5):e537-e544. doi: 10.1016/s2214$109 x(17) 30105-5$.

9. Foreman KJ, Lozano R, Lopez AD, Murray CJ. Modeling causes of death: an integrated approach using CODEm. Popul Health Metr. 2012;10:1. doi: 10.1186/1478-7954-10-1.

10. Mehdipour P, Navidi I, Parsaeian M, Mohammadi Y, Moradi Lakeh M, Rezaei Darzi E, et al. Application of Gaussian Process Regression (GPR) in estimating under-five mortality levels and trends in Iran 1990 - 2013, study protocol. Arch Iran Med. 2014;17(3):189-192.

11. Mehdipour P, Ghanbari A, Navidi I, Farzadfar F. Gaussian Process Regression for Mortality Rates. R Package version 0.1.0. 2018. Available from: https:/CRAN.R-project.org/ package $=$ GPRMortality.

12. Rajaratnam JK, Marcus JR, Flaxman AD, Wang H, Levin-Rector A, Dwyer L, et al. Neonatal, postneonatal, childhood, and under-5 mortality for 187 countries, 1970-2010: a systematic analysis of progress towards Millennium Development Goal 4. Lancet. 2010;375(9730):1988-2008. doi: 10.1016/S0140-6736(10)60703-9.

13. Forouzanfar MH, Alexander L, Anderson HR, Bachman VF, Biryukov S, Brauer $\mathrm{M}$, et al. Global, regional, and national comparative risk assessment of 79 behavioural, environmental and occupational, and metabolic risks or clusters of risks in 188 countries, 1990-2013: a systematic analysis for the Global Burden of Disease Study 2013. Lancet. 2015;386(10010):2287-2323. doi: 10.1016/ S0140-6736(15)00128-2.

14. Danaei G, Finucane MM, Lin JK, Singh GM, Paciorek CJ, Cowan MJ, et al. National, regional, and global trends in systolic blood pressure since 1980: systematic analysis of health examination surveys and epidemiological studies with 786 country-years and 5.4 million participants. Lancet. 2011;377(9765):568-77. doi: 10.1016/S01406736(10)62036-3.

15. Forouzanfar MH, Afshin A, Alexander LT, Anderson HR, Bhutta ZA, Biryukov S, et al. Global, regional, and national comparative risk assessment of 79 behavioural, environmental and occupational, and metabolic risks or clusters of risks, 1990-2015: a systematic analysis for the Global Burden of Disease Study 2015. Lancet. 2016;388(10053):1659-724. doi: 10.1016/s0140-6736(16)31679-8.
16. Lim SS, Vos T, Flaxman AD, Danaei G, Shibuya K, Adair-Rohani H, et al. A comparative risk assessment of burden of disease and injury attributable to 67 risk factors and risk factor clusters in 21 regions, 1990-2010: a systematic analysis for the Global Burden of Disease Study 2010. Lancet. 2012;380(9859):2224-60. doi: 10.1016/S01406736(12)61766-8.

17. Powles J, Fahimi S, Micha R, Khatibzadeh S, Shi P, Ezzati M, et al. Global, regional and national sodium intakes in 1990 and 2010: a systematic analysis of $24 \mathrm{~h}$ urinary sodium excretion and dietary surveys worldwide. BMJ Open. 2013;3(12):e003733. doi: 10.1136/ bmjopen-2013-003733.

18. Farzadfar F, Murray CJ, Gakidou E, Bossert T, Namdaritabar $\mathrm{H}$, Alikhani S, et al. Effectiveness of diabetes and hypertension management by rural primary health-care workers (Behvarz workers) in Iran: a nationally representative observational study. Lancet. 2012;379(9810):47-54. doi: 10.1016/S0140-6736(11)61349-4.

19. Whelton PK, Carey RM, Aronow WS, Casey DE Jr, Collins KJ, Dennison Himmelfarb C, et al. 2017 ACC/AHA/AAPA/ABC/ ACPM/AGS/APhA/ASH/ASPC/NMA/PCNA Guideline for the Prevention, Detection, Evaluation, and Management of High Blood Pressure in Adults: A Report of the American College of Cardiology/American Heart Association Task Force on Clinical Practice Guidelines. J Am Coll Cardiol. 2018;71(19):e127-e248. doi: 10.1016/j.jacc.2017.11.006.

20. Sepanlou SG, Kamangar F, Poustchi H, Malekzadeh R. Reducing the burden of chronic diseases: a neglected agenda in Iranian health care system, requiring a plan for action. Arch Iran Med. 2010;13(4):34050 .

21. Sepanlou SG, Poustchi H, Kamangar F, Malekzadeh R. Effectiveness and feasibility of lifestyle and low-cost pharmacologic interventions in the prevention of chronic diseases: a review. Arch Iran Med. 2011;14(1):46-53.

22. Farzadfar F, Danaei G, Namdaritabar H, Rajaratnam JK, Marcus JR, Khosravi A, et al. National and subnational mortality effects of metabolic risk factors and smoking in Iran: a comparative risk assessment. Popul Health Metr. 2011;9(1):55. doi: 10.1186/14787954-9-55.

23. Peykari N, Hashemi H, Dinarvand R, Haji-Aghajani M, Malekzadeh $\mathrm{R}$, Sadrolsadat A, et al. National action plan for non-communicable diseases prevention and control in Iran; a response to emerging epidemic. J Diabetes Metab Disord. 2017;16:3. doi: 10.1186/ s40200-017-0288-4. 